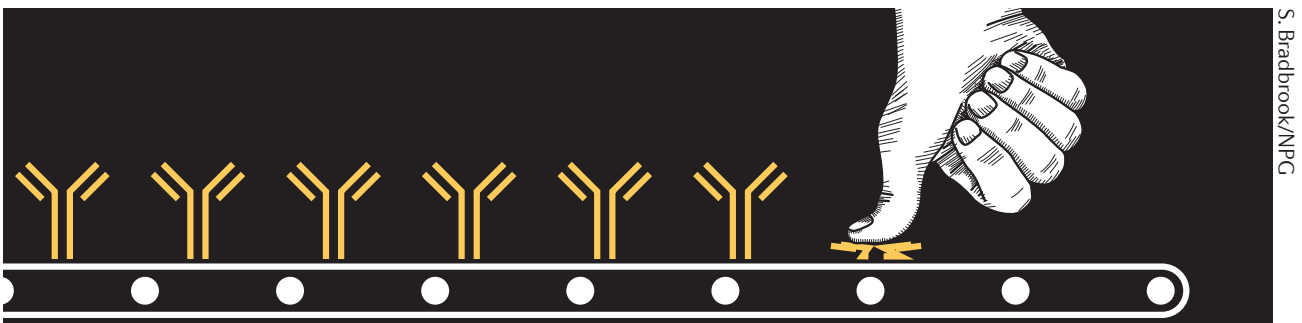

REGULATORY T CELLS

\title{
Suppressing antibody production
}

T follicular regulatory cells ( $\mathrm{T}_{\mathrm{FR}}$ cells) are a recently described subset of regulatory $T$ cells that specifically inhibits antibody production within germinal centres (GCs). Reporting in Nature Immunology, Sharpe and colleagues provide new insights into how $\mathrm{T}_{\mathrm{FR}}$ cells exert this suppressive effect.

The GC reaction is a highly regulated process that involves the interaction of $\mathrm{T}$ follicular helper cells $\left(T_{F H}\right.$ cells) with cognate B cells, during which $\mathrm{T}_{\mathrm{FH}}$ cells provide key signals - such as through interleukin-4 (IL-4), IL-21 and costimulatory molecules - for robust B cell activation, while the $B$ cells provide antigenic and costimulatory signals to the $T_{F H}$ cells. This reciprocal interaction drives class-switch recombination (CSR), somatic hypermutation and antibody production by the $B$ cells. It is known that $T_{F R}$ cells inhibit cytokine production by $T_{F H}$ cells and inhibit CSR and antibody production by $B$ cells, but the underlying mechanisms of this suppression are unknown.

To investigate how $T_{F R}$ cells inhibit the GC reaction, the authors developed an in vitro suppression assay in which cognate $T_{F H}$ cells and $B$ cells are cultured in the presence (suppressed cultures) or absence (activated cultures) of $T_{F R}$ cells. Although the early activation of B cells was unaffected, the addition of $T_{F R}$ cells resulted in reduced CSR and antibody production, and this suppression occurred in a cell contact-dependent manner. These data, combined with imaging studies, suggest that $T_{F R}$ cells physically disrupt the cognate interaction between $\mathrm{T}_{\mathrm{FH}}$ cells and $B$ cells during the $\mathrm{GC}$ reaction.

Next, the authors performed RNA-sequencing analysis on cells isolated from the activated and suppressed cultures. The expression of essential lineage transcription factors was unaffected, whereas the expression of the effector molecules Il4 and Il21 was markedly reduced in $\mathrm{T}_{\mathrm{FH}}$ cells from the suppressed cultures compared with $\mathrm{T}_{\mathrm{FH}}$ cells from the activated cultures. Similarly, suppressed $B$ cells retained their GC B cell transcriptional signature but showed reduced expression of genes encoding specific effector molecules. In both cell types the suppression state did not strongly resemble anergy or exhaustion.
Further investigation showed that the expression of genes associated with numerous metabolic pathways, including glycolysis, serine biosynthesis, purine metabolism, one-carbon metabolism and the tricarboxylic acid cycle, was greatly reduced in suppressed B cells. Inhibition of glycolysis or purine metabolism in activated $B$ cells robustly suppressed antibody production, which suggests that inhibiting these pathways can recapitulate the suppression of antibody production by $\mathrm{T}_{\mathrm{FR}}$ cells.

The culture of $B$ cells from suppressed cultures in a second culture with just $T_{F H}$ cells showed that the $B$ cells retained their defects in CSR, antibody production and metabolism, which suggests that $B$ cell suppression can persist in the absence of $T_{F R}$ cells. These $B$ cells showed evidence of chromatin inaccessibility at genes encoding products important for B cell function, which may explain, at least in part, the durable suppressive effect of $T_{F R}$ cells on GC B cells.

Importantly, the addition of IL-21 to the suppressed cultures blocked the suppression of $B$ cell antibody production and metabolism by $T_{F R}$ cells. This 'rescuing' effect of IL-21 in suppressed cultures was mediated in part by enhancing glycolysis in B cells, and the restoration of CSR and antibody production required signalling through the IL-21 receptor on $B$ cells. Additional data suggested that IL-21 might also be able to alter $T_{F R}$ cell metabolism and thereby reduce the suppressive capacity of this regulatory subset.

Together, these data show that $\mathrm{T}_{\mathrm{FR}}$ cells induce a distinct suppressive state in $\mathrm{T}_{\mathrm{FH}}$ cells and $B$ cells to inhibit antibody production. This $T_{F R}$ cell-mediated suppressive effect can be overcome by exogenous IL-21, providing a possible mechanism to circumvent suppression by $T_{F R}$ cells and modulate antibody production in vivo.

\section{Olive Leavy}

ORIGINAL ARTICLE Sage, P. T. et al. Suppression by $T_{\mathrm{FR}}$ cells leads to durable and selective inhibition of B cell effector function. Nat. Immunol. http://dx.doi.org/10.1038/ni.3578 (2016) FURTHER READING Qi, H. T follicular helper cells in space-time. Nat. Rev. Immunol. 16, 612-625 (2016)
This 'rescuing' effect of IL-21

in suppressed cultures was mediated in part by enhancing glycolysis in B cells 